Research Article

\title{
G-Expansibility and G-Almost Periodic Point under Topological Group Action
}

\author{
Zhanjiang Ji \\ School of Data Science and Software Engineering, Wuzhou University, Wuzhou 543002, Guangxi, China \\ Correspondence should be addressed to Zhanjiang Ji; jizhanjiang1985@126.com
}

Received 21 June 2021; Accepted 18 October 2021; Published 8 November 2021

Academic Editor: José Francisco Gómez Aguilar

Copyright $\odot 2021$ Zhanjiang Ji. This is an open access article distributed under the Creative Commons Attribution License, which permits unrestricted use, distribution, and reproduction in any medium, provided the original work is properly cited.

Firstly, the new concepts of $G$-expansibility, $G$-almost periodic point, and $G$-limit shadowing property were introduced according to the concepts of expansibility, almost periodic point, and limit shadowing property in this paper. Secondly, we studied their dynamical relationship between the self-map $f$ and the shift map $\sigma$ in the inverse limit space under topological group action. The following new results are obtained. Let $(X, d)$ be a metric $G$-space and $(X, \bar{G}, \bar{d}, \sigma)$ be the inverse limit space of $(X, G, d, f)$. (1) If the map $f: X \longrightarrow X$ is an equivalent map, then we have $A P_{\bar{G}}(\sigma)=\operatorname{Lim}\left(A p_{G}(f), f\right)$. (2) If the map $f: X \longrightarrow X$ is an equivalent surjection, then the self-map $f$ is $G$-expansive if and only if the shift map $\sigma$ is $\bar{G}$-expansive. (3) If the map $f: X \longrightarrow X$ is an equivalent surjection, then the self-map $f$ has $G$ - limit shadowing property if and only if the shift map $\sigma$ has $\bar{G}-$ limit shadowing property. The conclusions of this paper generalize the corresponding results given in the study by Li, Niu, and Liang and $\mathrm{Li}$. Most importantly, it provided the theoretical basis and scientific foundation for the application of tracking property in computational mathematics and biological mathematics.

\section{Introduction}

Let $(X, d)$ be a metric space and $f$ be a continuous map from $X$ to $X$. A point $x$ is called to be an almost periodic point if for each open set $U$ containing $x$ there exists a positive integer $m>0$ such that for every positive integer $k>0$ there exists $r \in(k, k+m]$ satisfying $f^{r}(x) \in U$ (see [1]). In recent years, there are many achievements about almost periodic points set (see [1-8]). Qiu and Zhao [2] proved that if the map $f$ has shadowing property in $X$, then the map $f$ has shadowing property in $\overline{A P}(f)$. Liao and Wang [3] discussed the properties of almost periodic points in unilateral symbol space. In [1], it is proved that the set of almost periodic points of shift map $\sigma$ is the inverse limit space formed by the self-map $f$ in $A P(f)$. Inspired by the idea of Li [1], the author gave the concepts of $G$-almost periodic point according to the concept of almost periodic point. That is, let $(X, d)$ be a metric $G$-space and $f$ be a continuous map from $X$ to $X$. A point $x$ is called to be an $G$-almost periodic point if for each open set $U$ containing $x$ there exists a positive integer $m>0$ such that for every positive integer $k>0$ there exists $r \in(k, k+m]$ and $g_{k} \in G$ satisfying $g_{k} f^{r}(x) \in U$. Next, we give the following theorem in the inverse limit space under topological group action.

Theorem 1. Let $(X, d)$ be a metric $G$-space, the map $f: X \longrightarrow X$ be an equivalent map, and $\left(X_{f}, \bar{G}, \bar{d}, \sigma\right)$ be the inverse limit space of $(X, G, d, f)$. Then, we have

$$
A P_{\bar{G}}(\sigma)=\operatorname{Lim}_{\leftarrow}\left(A p_{G}(f), f\right) .
$$

According to definition, $G$-almost periodic point means almost periodic point. Otherwise, it does not hold. Hence, Theorem 1 generalizes the corresponding results given in $\mathrm{Li}$ [1].

The map $f$ is said to be expansive if for any $x \neq y \in X$ there exists a positive integer $n>0$ such that $d\left(f^{n}(x), f^{n}(y)\right)>C$ (see [9]). The map $f$ has shadowing property if each $\varepsilon>0$ there exists $\delta>0$ such that for any 
$\delta$-pseudo orbit of $f$ there exists a point $y$ in $X$ such that the sequence $\left\{x_{i}\right\}_{i=0}^{\infty}$ is $\mathcal{E}$-shadowed by the point $y$ (see [10]). Expansibility and shadowing property have attracted the attention of many scholars. The relevant results are seen in [11-17]. Das and Das [11] pointed out that the map $f$ is $G$-expansive if and only if the map $g$ is $\bar{G}$-expansive in locally equidistant covered space. Wang and Zeng [12] discussed the relationship between average shadowing property and $q$-average shadowing property. Das [13] proved the nonexistence of pseudo equivariant $G$-expansive homeomorphism on closed unit interval. Das and Das [14] obtained a sufficient condition for the extension of a $G$-expansive homeomorphism on a G-invariant subspace of a compact metric $G$-space with $G$ compact to be $G$-expansive on the whole space. Shah [15] gave a necessary and sufficient condition for a positively $G$-expansive map to possess the $G$-shadowing property. Wu [16] proved that the system with $d$-tracking property is chain mixed. Oprocha et al. [17] analyzed necessary and sufficient conditions for shadowing property over a set with positive density. In this paper, we gave the concepts of $G$-expansive map and $G$-limit shadowing property according to the concepts of expansive map and limit shadowing property. At last, we will give the proof of Theorems 2 and 3 in the inverse limit space under topological group action. The main results are as follows.

Theorem 2. Let $(X, d)$ be a compact metric $G$-space, the map $f: X \longrightarrow X$ be an equivalent surjection, and $\left(X_{f}, \bar{G}, \bar{d}, \sigma\right)$ be the inverse limit space of $(X, G, d, f)$. Then, the self-map $f$ is $G$-expansive if and only if the shift map $\sigma$ is $\bar{G}$-expansive.

Theorem 3. Let $(X, d)$ be a compact metric $G$-space, the map $f: X \longrightarrow X$ be an equivalent surjection, and $\left(X_{f}, \bar{G}, \bar{d}, \sigma\right)$ be the inverse limit space of $(X, G, d, f)$. Then, the self-map $f$ has $G$-limit shadowing property if and only if the shift map $\sigma$ has $\bar{G}$-limit shadowing property.

According to definition, G-expansibility means expansibility and $G$-limit shadowing property means limit shadowing property. Otherwise, it does not hold. Hence, Theorems 2 and 3, respectively, generalize the corresponding results given in Niu [18] and Liang and Li [10].

\section{G-Almost Periodic Point under Topological Group Action}

Definition 1 (see [19]). Let $(X, d)$ be a metric $G$-space, $G$ be a topological group, and $\varphi: G \times X \longrightarrow X$ be a continuous map. The triple $(X, G, \theta)$ or $X$ is called to be metric $G$-space if the following conditions are satisfied:

(1) $\varphi(e, x)=x$ where $e$ is the identity of $G$ and for all $x \in X$

(2) $\varphi\left(g_{1}, \varphi\left(g_{2}, x\right)\right)=\varphi\left(g_{1} g_{2}, x\right)$ for all $x \in X$ and all $g_{1}$, $g_{2} \in G$
Remark 1. If $X$ is compact, then $X$ is also said to be compact metric $G$-space. For the convenience of writing, $\varphi(g, x)$ is usually abbreviated as $g x$.

Definition 2 (see [19]). Let $(X, d)$ be a metric $G$-space and $f$ be a continuous map from $X$ to $X . f$ is said to be an equivariant map if we have $f(p x)=p f(x)$ for all $x \in X$ and all $p \in G$.

Definition 3 (see [20]). Let $(X, d)$ be a metric space and $f$ be a continuous map from $X$ to $X . X_{f}$ is said to be the inverse limit spaces of $X$ if we write

$$
X_{f}=\left\{\left(x_{0}, x_{1}, x_{2}, \ldots\right): f\left(x_{i+1}\right)=x_{i}, \forall i \geq 0\right\} .
$$

The metric $\bar{d}$ in $X_{f}$ is defined by

$$
\bar{d}(\bar{x}, \bar{y})=\sum_{i=0}^{\infty} \frac{d\left(x_{i}, y_{i}\right)}{2^{i}}
$$

where $\bar{x}=\left(x_{0}, x_{1}, x_{2}, \ldots\right) \in X_{f} \quad$ and $\quad \bar{y}=\left(y_{0}, y_{1}, y_{2}\right.$, ...) $\in X_{f}$.

The shift map $\sigma: X_{f} \longrightarrow X_{f}$ is defined by

$$
\sigma(\bar{x})=\left(f\left(x_{0}\right), x_{0}, x_{1}, \ldots\right) .
$$

The projection map $\pi_{i}: X_{f} \longrightarrow X$ is defined by

$$
\pi_{i}(\bar{x})=x_{i} .
$$

Thus, $\left(X_{f}, \bar{d}\right)$ is compact metric space. The shift mapping $\sigma$ is homeomorphism, and for any $i \geq 0$, the projection map $\pi_{i}$ is a continuous and open map.

Definition 4 (see [20]). Let $(X, d)$ be a metric G-space and $f$ be equivariant map from $X$ to $X$. Write

$$
\begin{aligned}
\bar{G} & =\{(g, g, g, \ldots): g \in G\}, \\
G_{\infty} & =\prod_{i=0}^{\infty} G_{i},
\end{aligned}
$$

where $G_{i}=G$.

The map $\theta: \bar{G} \times X_{f} \longrightarrow X_{f}$ is defined by

$$
\theta(\bar{g}, \bar{x})=\bar{g} \cdot \bar{x}=\left(g x_{0}, g x_{1}, g x_{2}, \ldots\right),
$$

where $\bar{g}=(g, g, g, \ldots) \in \bar{G}$ and $\bar{x}=\left(x_{0}, x_{1}, x_{2}, \ldots\right) \in X_{f}$.

Then, $\left(X_{f}, \bar{G}, \theta\right)$ is a metric $G$-space. Let $(X, G, d, f)$ and $\left(X_{f}, \bar{G}, \bar{d}, \sigma\right)$ be shown as above. The space $\left(X_{f}, \bar{G}, \bar{d}, \sigma\right)$ is called to be the inverse limit spaces of $(X, d, f, G)$ under group action.

Definition 5 (see [1]). Let $(X, d)$ be a metric space and $f$ be a continuous map from $X$ to $X$. The point $x$ is called to be an almost periodic point if for each open set $U$ containing $x$ there exists a positive integer $m>0$ such that for every positive integer $k>0$ there exists $r \in(k, k+m]$ satisfying $f^{r}(x) \in U$. Denoted by $A P(f)$ the almost periodic point set of the map $f$. 
Remark 2. According to the concept of almost periodic point, we give the concept of $G$-almost periodic point.

Definition 6. Let $(X, d)$ be a metric space and $f$ be a continuous map from $X$ to $X$. The point $x$ is called to be an $G$-almost periodic point if for each open set $U$ containing $x$ there exists a positive integer $m>0$ such that for every positive integer $k>0$ there exists $r \in(k, k+m]$ and $g_{k} \in G$ satisfying $g_{k} f^{r}(x) \in U$. Denoted by $A P_{G}(f)$ the $G$-almost periodic point set of the map $f$.

Theorem 4. Let $(X, d)$ be a metric $G$-space, the map $f: X \longrightarrow X$ be an equivalent map, and $\left(X_{f}, \bar{G}, \bar{d}, \sigma\right)$ be the inverse limit space of $(X, G, d, f)$. Then, we have

$$
A P_{\bar{G}}(\sigma)=\operatorname{Lim}_{\leftarrow}\left(A p_{G}(f), f\right) .
$$

Proof. $\Longrightarrow$ Suppose $\bar{x}=\left(x_{0}, x_{1}, x_{2}, \ldots\right) \in A P_{\bar{G}}(\sigma)$. For any $i \geq 0$, let $U$ be any open set containing $x_{i}$. Then, $\pi_{i}^{-1}(U)$ is an open set containing $\bar{x}$. Hence, there exists a positive integer $m_{1}>0$ such that for every positive integer $k>0$ there exists $p \in\left(k, k+m_{1}\right]$ and $\bar{g}_{k}=\left(g_{k}, g_{k}, g_{k}, \ldots\right) \in \bar{G}$ such that

$$
\bar{g}_{k} \sigma^{p}(\bar{x}) \in \pi_{i}^{-1}(U)
$$

Thus, we can obtain

$$
\pi_{i}\left(\bar{g}_{k} \sigma^{p}(\bar{x})\right) \in U
$$

Then, we have that

$$
g_{k} f^{p}\left(x_{i}\right) \in U
$$

So, $x_{i} \in A P_{G}(f)$. Hence, $A P_{\bar{G}}(\sigma) \subset \operatorname{Lim}\left(A P_{G}(f), f\right)$.

$\Longleftarrow$ Suppose $\bar{y}=\left(y_{0}, y_{1}, y_{2}, \ldots\right) \in \operatorname{Lim}_{\leftarrow}\left(A P_{G}(f), f\right)$. Thus, we can get $y_{i} \in A P_{G}(f)$ for every $i \geq 0$. Let $V$ be an any open set containing the point $\bar{y}$. Then, $\pi_{i}(V)$ is an open set containing the point $y_{i}$. According to $y_{i} \in A P_{G}(f)$, there exists a positive integer $m_{2}>0$ such that for every positive integer $n>0$ there exists $r \in\left(n, n+m_{2}\right]$ and $t_{n} \in G$ satisfying

$$
t_{n} f^{r}\left(y_{i}\right) \in \pi_{i}(V), \quad i \geq 0 \text {. }
$$

Let $\bar{t}_{n}=\left(t_{n}, t_{n}, t_{n}, \ldots\right) \in \bar{G}$. Then, we can obtain

$$
\bar{t}_{n} \sigma^{r}(\bar{y}) \in V \text {. }
$$

So, $\quad \bar{y} \in A P_{\bar{G}}(\sigma)$. Thus, we have $\operatorname{Lim}\left(A P_{G}(f), f\right) \subset A P_{\bar{G}}(\sigma)$. This completes the proof.

\section{G-Expansibility under Topological Group Action}

Definition 7 (see [9]). Let $(X, d)$ be a metric space, $f$ be a continuous map from $X$ to $X$, and $C$ be a positive constant. The map $f$ is said to be an expansive map if for any $x \neq y \in X$ there exists a positive integer $n>0$ such that $d\left(f^{n}(x), f^{n}(y)\right)>C$ where the constant $C$ is called to be an expansion constant.
Remark 3. According to the concept of expansive map, we give the concept of $G$-almost expansive map.

Definition 8. Let $(X, d)$ be a metric $G$-space, $f$ be a continuous map from $X$ to $X$, and $C$ be a positive constant. The map $f$ is said to be an $G$-expansive map if for any $x \neq y \in X$ there exists an positive integer $n$ such that for any $g$ and $p \in G$ we have that $d\left(f^{n}(g x), f^{n}(p y)\right)>C$ where the constant $C$ is called to be an $G$-expansion constant.

Next, we start to prove Theorem 5 .

Theorem 5. Let $(X, d)$ be a compact metric $G$-space, the map $f: X \longrightarrow X$ be an equivalent surjection, and $\left(X_{f}, \bar{G}, \bar{d}, \sigma\right)$ be the inverse limit space of $(X, G, d, f)$. Then, the self-map $f$ is $G$-expansive if and only if the shift map $\sigma$ is $\bar{G}$-expansive.

Proof. $\Longrightarrow$ Suppose that the self-map $f$ is $G$-expansive map with expansion constant $C_{1}>0$. Thus, the map $f$ is injective. Let $\bar{x}=\left(x_{0}, x_{1}, x_{2}, \ldots\right) \in X_{f}$ and $\bar{y}=\left(y_{0}, y_{1}, y_{2}, \ldots\right) \in X_{f}$ with $\bar{x} \neq \bar{y}$. As the map $f$ is injective, we can get $x_{0} \neq y_{0}$. Let

$$
\begin{aligned}
\bar{g} & =(g, g, g, \ldots) \in \bar{G}, \\
\bar{l} & =(l, l, l, \ldots) \in \bar{G} .
\end{aligned}
$$

By the definition of $G$-expansive map $f$, there exists an positive integer $n_{1}>0$ such that

$$
d\left(f^{n_{1}}\left(g x_{0}\right), f^{n_{1}}\left(l y_{0}\right)\right)>C_{1} .
$$

By the definition of the metric $\bar{d}$, we get that

$$
\bar{d}\left(\sigma^{n_{1}}(\bar{g} \cdot \bar{x}), \sigma^{n_{1}}(\bar{l} \cdot \bar{y})\right)>C_{1} .
$$

Hence, the shift map $\sigma$ is $\bar{G}$-expansive.

$\Longleftarrow$ Suppose that the shift map $\sigma$ is $\bar{G}$-expansive map with expansion constant $4 C_{2}>0$. Since $X$ is compact metric space, it is bounded. Write $M=\operatorname{Diam}(X)$. Let $m>0$ such that

$$
\sum_{i=m+1}^{\infty} \frac{M}{2^{i}}<2 C_{2} .
$$

Since the map $f$ is surjection, for any $u \neq v \in X$, we can choose

$$
\begin{aligned}
& \bar{u}=\left(f^{m}(u), f^{m-1}(u), \ldots, f(u), u, \ldots\right) \in X_{f}, \\
& \bar{v}=\left(f^{m}(v), f^{m-1}(v), \ldots f(v), v, \ldots\right) \in X_{f} .
\end{aligned}
$$

It is obvious that the point $\bar{u}$ is different from the point $\bar{v}$. For any $s$ and $t \in G$, let

$$
\begin{aligned}
& \bar{s}=(s, s, s, \ldots) \in \bar{G}, \\
& \bar{t}=(t, t, t, \ldots) \in \bar{G} .
\end{aligned}
$$

According to that the shift map $\sigma$ is $\bar{G}$-expansive map with expansion constant $4 C_{2}>0$, there exist a positive integer $n_{2}>0$ such that

$$
\bar{d}\left(\sigma^{n_{2}}(\bar{s} \cdot \bar{u}), \sigma^{n_{2}}(\bar{t} \cdot \bar{v})\right)>4 C_{2} .
$$


According to that the map $f$ is equivalent, we can get that

$$
\sum_{i=0}^{m} \frac{d\left(s f^{n_{2}+m-i}(u), t f^{n_{2}+m-i}(v)\right)}{2^{i}}+\sum_{i=m+1}^{\infty} \frac{M}{2^{i}}>4 C_{2}
$$

By (17), we have that

$$
\sum_{i=0}^{m} \frac{d\left(s f^{n_{2}+m-i}(u), t f^{n_{2}+m-i}(v)\right)}{2^{i}}>2 C_{2} .
$$

If for any positive integer $k>0$, we have that

$$
d\left(s f^{k}(u), t f^{k}(v)\right) \leq C_{2} .
$$

Then, by (23), we can get that

$$
\sum_{i=0}^{m} \frac{d\left(s f^{n_{2}+m-i}(u), t f^{n_{2}+m-i}(v)\right)}{2^{i}} \leq \sum_{i=0}^{m} \frac{C_{2}}{2^{i}}<2 C_{2} .
$$
that

It is absurd. Hence, there exists a positive integer $n_{3}$ such

$$
d\left(s f^{n_{3}}(u), t f^{n_{3}}(v)\right)>C_{2} .
$$

So, the self-map $f$ is $G$-expansive. Thus, we end the proof.

\section{G-Limit Shadowing Property under Topological Group Action}

Definition 9 (see [21]). Let $(X, d)$ be a metric $G$-space and $f$ be a continuous map from $X$ to $X$. The sequence $\left\{x_{i}\right\} i \geq 0$ is called to be $G$-limit pseudo orbit of $f$ if for any $i \geq 0$ there exists $t_{i} \in G$ such that $\lim _{i \rightarrow \infty} d\left(t_{i} f\left(x_{i}\right), x_{i+1}\right)=0$.

Definition 10 (see [21]). Let $(X, d)$ be a metric $G$-space and $f$ be a continuous map from $X$ to $X .\left\{x_{i}\right\} i \geq 0$ is said to be $G$-limit shadowed by a point $y$ if for any $i \geq 0$ there exists $t_{i} \in G$ such that $\lim _{i \longrightarrow \infty} d\left(f^{i}(y), t_{i} x_{i}\right)=0$.

Definition 11 (see [21]). Let $(X, d)$ be a metric $G$-space and $f$ be a continuous map from $X$ to $X$.

The map $f$ has $G$-limit shadowing property if for any $G$-limit pseudo orbit $\left\{x_{i}\right\}_{i=0}^{\infty}$ of $f$ there exists a point $y$ in $X$ such that the sequence $\left\{x_{i}\right\}_{i=0}^{\infty}$ is $G$-limit shadowed by the point $y$.

The main result of this section is the following theorem:

Theorem 6. Let $(X, d)$ be a compact metric G-space, the map $f: X \longrightarrow X$ be an equivalent surjection, and $\left(X_{f}, \bar{G}, \bar{d}, \sigma\right)$ be the inverse limit space of $(X, G, d, f)$. Then, the self-map $f$ has $G$-limit shadowing property if and only if the shift map $\sigma$ has $\bar{G}$-limit shadowing property.

Proof. $\Longrightarrow$ Suppose that the self-map $f$ has $G$-limit shadowing property. Since $X$ is compact, it is bounded. Write $M=\operatorname{Diam}(X)$. For any $\varepsilon>0$, let $m>0$ such that

$$
\frac{M}{2^{m}}<\frac{\varepsilon}{2}
$$

By the continuity of the map $f$, it follows that for given $(\varepsilon / 4)>0$ and any $0 \leq i \leq m$ there exists $0<\delta_{1}<(\varepsilon / 4)$ such that $d(x, y)<\delta_{1}$ which implies

$$
d\left(f^{i}(x), f^{i}(y)\right)<\frac{\varepsilon}{4} .
$$

Let $\left\{\bar{y}_{n}\right\}_{n=0}^{\infty}$ be $\bar{G}$-limit pseudo orbit of the shift map $\sigma$ with $\bar{y}_{n}=\left(y_{n}^{0}, y_{n}^{1}, y_{n}^{2}, \ldots\right) \in X_{f}$. Then, there exists $\bar{g}_{n} \in \bar{G}$ with $\bar{g}_{n}=\left(g_{n}, g_{n}, g_{n}, \ldots\right) \in \bar{G}$ such that

$$
\lim _{n \longrightarrow \infty} \bar{d}\left(\bar{g}_{n} \sigma\left(\bar{y}_{n}\right), \bar{y}_{n+1}\right)=0 .
$$

Hence, there exists a positive integer $N_{1} \in N_{+}$such that when $n>N_{1}$, we have that

$$
\bar{d}\left(\bar{g}_{n} \sigma\left(\bar{y}_{n}\right), \bar{y}_{n+1}\right)<\frac{\varepsilon}{2^{m}} .
$$

So, when $n>N_{1}$, we have that

$$
d\left(g_{n}^{m} f\left(y_{n}^{m}\right), y_{n+1}^{m}\right)<\varepsilon .
$$

Thus, we can get that

$$
\lim _{n \longrightarrow \infty} d\left(g_{n}^{m} f\left(y_{n}^{m}\right), y_{n+1}^{m}\right)=0 .
$$

Hence, $\left\{y_{n}^{m}\right\}_{n=0}^{\infty}$ is $G$-limit pseudo orbit of the map $f$. According to the hypothesis, for any $n \geq 0$, there exist $z \in X$ and $t_{n} \in G$ such that

$$
\lim _{n \longrightarrow \infty} d\left(f^{n}(z), t_{n} y_{n}^{m}\right)=0 .
$$

So, there exists a positive integer $N_{2} \in N_{+}$such that when $n>N_{2}$, we have that

$$
d\left(f^{n}(z), t_{n} y_{n}^{m}\right)<\delta_{1} .
$$

According to the equivalent definition of the map $f$ and (27), for any $0 \leq i \leq m$, we have that

$$
d\left(f^{n+i}(z), t_{n} y_{n}^{m-i}\right)<\frac{\varepsilon}{4}
$$

Since the map $f$ is onto, we can choose

$$
\begin{aligned}
\bar{z} & =\left(f^{m}(z), f^{m-1}(z), f^{m-2}(z), \ldots, f(z), z \ldots\right) \in X_{f}, \\
\bar{t}_{n} & =\left(t_{n}, t_{n}, t_{n}, \ldots, t_{n}, \ldots\right) \in \bar{G} .
\end{aligned}
$$

By (26) and (34), when $n>N_{2}$, we have that

$$
\bar{d}\left(\sigma^{n}(\bar{z}), \bar{t}_{n} \bar{y}_{n}\right)<\sum_{i=0}^{m} \frac{1}{2^{i}} \cdot \frac{\varepsilon}{4}+\frac{M}{2^{m}}<\varepsilon .
$$

Hence, we can get that

$$
\lim _{n \longrightarrow \infty} \bar{d}\left(\sigma^{n}(\bar{z}), \bar{t}_{n} \bar{y}_{n}\right)=0
$$


So, the shift map $\sigma$ has $\bar{G}$-limit shadowing property.

$\Longleftarrow$ Suppose that the shift map $\sigma$ has $\bar{G}$-limit shadowing property. For any $\eta>0$, there exists an positive integer $m^{\prime}>0$ such that

$$
\frac{M}{2^{m^{\prime}}}<\frac{\eta}{2}
$$

According to the continuity of the map $f$, it follows that for given $(\eta / 4)>0$ and any $0 \leq i \leq m^{\prime}$ there exists $0<\delta_{2}<(\eta / 4)$ such that $d(x, y)<\delta_{2}$ implies

$$
d\left(f^{i}(x), f^{i}(y)\right)<\frac{\eta}{4} .
$$

Let $\left\{x_{k}\right\}_{k=0}^{\infty}$ be $G$-limit pseudo orbit of the map $f$. Then, for any $k \geq 0$, there exists $t_{k} \in G$ such that

$$
\lim _{k \rightarrow \infty} d\left(t_{k} f\left(x_{k}\right), x_{k+1}\right)=0 .
$$

Hence, there exists a positive integer $N_{3} \in N_{+}$such that when $k>N_{3}$, we have that

$$
d\left(t_{k} f\left(x_{k}\right), x_{k+1}\right)<\delta_{3} .
$$

According to the equivalent definition of the map $f$ and (39), for any $0 \leq i \leq m^{\prime}$, we have that

$$
d\left(t_{k} f^{i+1}\left(x_{k}\right), f^{i}\left(x_{k+1}\right)\right)<\frac{\eta}{4} \text {. }
$$

Since the map $f$ is onto, we can write

$$
\begin{aligned}
\bar{x}_{k} & =\left(f^{m^{\prime}}\left(x_{k}\right), f^{m^{\prime}-1}\left(x_{k}\right), f^{m^{\prime}-2}\left(x_{k}\right), \ldots, f\left(x_{k}\right), x_{k}, \ldots\right) \in X_{f}, \\
\bar{t}_{k} & =\left(t_{k}, t_{k}, t_{k}, \ldots, t_{k}, \ldots\right) \in \bar{G} .
\end{aligned}
$$

By (38) and (42), when $k>N_{3}$, we have that

$$
\bar{d}\left(\bar{t}_{k} \sigma\left(\bar{x}_{k}\right), \bar{x}_{k+1}\right)<\sum_{i=0}^{m} \frac{1}{2^{i}} \cdot \frac{\eta}{4}+\frac{M}{2^{m}}<\eta .
$$

Hence, we can get that

$$
\lim _{k \longrightarrow \infty} \bar{d}\left(\bar{t}_{k} \sigma\left(\bar{x}_{k}\right), \bar{x}_{k+1}\right)=0 .
$$

Thus, $\left\{\bar{x}_{k}\right\}_{k=0}^{\infty}$ is $\bar{G}$-limit pseudo orbit of the shift map $\sigma$. By the hypothesis, for any $k \geq 0$, there exist $\bar{s}=\left(s_{0}, s_{1}, s_{2}, \ldots\right) \in X_{f}$ and $\bar{p}_{k}=\left(p_{k}, p_{k}, p_{k}, \ldots\right) \in \bar{G}$ such that

$$
\lim _{k \longrightarrow \infty} \bar{d}\left(\sigma^{k}(\bar{s}), \bar{p}_{k} \cdot \bar{x}_{k}\right)=0 .
$$

Hence, there exists a positive integer $N_{4} \in N_{+}$such that when $k>N_{4}$, we have that

$$
\bar{d}\left(\sigma^{k}(\bar{s}), \bar{p}_{k} \cdot \bar{x}_{k}\right)<\frac{\eta}{2^{m^{\prime}}} .
$$

So, when $k>N_{4}$, we have that

$$
d\left(f^{k}\left(s_{m^{\prime}}\right), p_{k} \cdot x_{k}\right)<\eta
$$

Thus, we have that

$$
\lim _{k \rightarrow \infty} d\left(f^{k}\left(s_{m^{\prime}}\right), p_{k} x_{k}\right)=0
$$

Hence, the self-map $f$ has $G$-limit shadowing property. This completes the proof.

\section{Conclusion}

Firstly, the new concepts of $G$-expansibility, $G$-almost periodic point, and $G$-limit shadowing property were introduced according to the concepts of expansibility, almost periodic point, and limit shadowing property in this paper. Secondly, we studied their dynamical relationship between the self-map $f$ and the shift map $\sigma$ in the inverse limit space under topological group action. The following new results are obtained in the inverse limit space under topological group action. (1) If the map $f: X \longrightarrow X$ is an equivalent map, then we have $A P_{\bar{G}}(\sigma)=\operatorname{Lim}\left(A p_{G}(f), f\right)$. (2) If the map $f: X \longrightarrow X$ is an equivalent surjection, then the selfmap $f$ is $G$-expansive if and only if the shift map $\sigma$ is $\bar{G}$-expansive. (3) If the map $f: X \longrightarrow X$ is an equivalent surjection, then the self-map $f$ has $G$-limit shadowing property if and only if the shift map $\sigma$ has $\bar{G}$-limit shadowing property.

$G$-almost periodic point, $G$-expansibility, and $G$-limit shadowing property, respectively, mean almost periodic point, expansibility, and limit shadowing property. Otherwise, it does not hold. Hence, Theorems 1-3, respectively, generalize the corresponding results given in the study by $\mathrm{Li}$ [1], Niu [18], and Liang and Li [10]. Most importantly, it provided the theoretical basis and scientific foundation for the application of tracking property in computational mathematics and biological mathematics.

\section{Data Availability}

The data used to support the findings of this study are included in references [1-21] in the article.

\section{Conflicts of Interest}

The author declares that there are no conflicts of interest regarding the publication of this article.

\section{Acknowledgments}

This research was partially supported by the NSF of Guangxi Province (2020JJA110021) and construction project of Wuzhou University of China (2020B007).

\section{References}

[1] S. H. Li, "Dynamical properties of the shift maps on the inverse limit spaces," Ergodic Theory and Dynamical Systems, vol. 12, pp. 95-108, 1992.

[2] Y. Qiu and J. L. Zhao, "Pseudo orbit tracking property and almost periodic points," Journal of Guangxi Normal University, vol. 25, pp. 50-53, 2007. 
[3] G. F. Liao and L. D. Wang, "Almost periodicity and SS scrambled set," Chinese Annals of Mathematics, vol. 23, pp. 685-692, 2002.

[4] L. D. Wang, N. Li, and H. Wang, "Complexity and proper quasi-weakly almost periodic points," Chaos, Solitons \& Fractals, vol. 139, pp. 25-34, 2020.

[5] A. Artigue, "Local stable sets of almost periodic points," Topology and its Applications, vol. 272, pp. 27-36, 2020.

[6] Y. Qi and D. Y. Jian, "On quasi-weakly almost periodic points of continuous flows," Acta Mathematica Sinica, vol. 35, pp. 257-269, 2019.

[7] B. Ling, X. X. Nie, and J. D. Yin, "Weak almost periodic points and chaotic dynamics of discrete amenable group actions," Journal of the Korean Mathematical Society, vol. 56, pp. 39-52, 2019.

[8] Y. Qi, D. Y. Jian, and W. Tao, "A note on quasi-weakly almost periodic point," Acta Mathematica Sinica, vol. 31, pp. 637646, 2015.

[9] B. F. Bryant, "Expansive self-homeomorphisms of a compact metric space," The American Mathematical Monthly, vol. 69, pp. 386-391, 1962.

[10] C. Liang and S. H. Li, "Shadowing property for inverse limit spaces," Proceedings of the American Mathematical Society, vol. 115, pp. 573-580, 1992.

[11] R. Das and T. Das, "On properties of G-expansive homeomorphisms," Mathematica Slovaca, vol. 62, pp. 531-538, 2012.

[12] H. Y. Wang and P. Zeng, "Partial shadowing of averagepseudo-orbits," Scientia Sinica (Mathematica), vol. 46, pp. 781-792, 2016.

[13] R. Das, "On G-expansive homeomorphisms and generators," Journal of the Indian Mathematical Society, vol. 72, pp. 83-89, 2005.

[14] R. Das and T. K. Das, "On extension of G-expansive homeomorphisms," Journal of the Indian Mathematical Society, vol. 67, pp. 35-41, 2000.

[15] E. Shah, "Positively expansive maps on G-spaces," Journal of the Indian Mathematical Society, vol. 72, pp. 91-97, 2005.

[16] X. X. Wu, "Some remarks on d-shadowing property," Scientia Sinica (Mathematica), vol. 45, pp. 273-286, 2015.

[17] P. Oprocha, D. A. Dastjerdi, and M. Hosseini, "On partial shadowing of complete pseudo-orbits," Journal of Mathematical Analysis and Applications, vol. 404, pp. 47-56, 2013.

[18] Y.X. Niu, "The shift maps on the inverse limit spaces," Journal of Anqing Normal University (Natural Science Edition), vol. 8, pp. 1-2, 2002.

[19] E. Shah and T. Das, "Consequences of shadowing property of G-Spaces," International Journal of Mathematical Analysis, vol. 7, pp. 579-588, 2013.

[20] Z. J. Ji, Dynamical Property of Product Space and the Inverse Limit Space of a Topological Group Action, Guangxi University, Nanning, China, 2014.

[21] Z. J. Ji and W. Shi, "The research on strong shadowing property and limit shadowing property in product metric G-space," Journal of Inner Mongolia Normal University (Natural Science Edition), vol. 49, pp. 189-193, 2020. 